\title{
Chip-to-package Wireless Power Transfer and its Application to mm-Wave Antennas and Monolithic Radiometric Receivers
}

\author{
Luca Aluigi ${ }^{\#, *}$, Trang T. Thai ${ }^{*}$, Manos M. Tentzeris ${ }^{*}$, Luca Roselli" and Federico Alimenti\# \\ \# DIEI, University of Perugia, Via Duranti 93, 06125 Perugia, Italy \\ *GEDEC, Georgia Institute of Technology, Atlanta, 30332-0250, USA
}

\begin{abstract}
A chip-to-package wireless power transfer concept is applied to MMIC and antennas on LCP substrate is presented. Electromagnetic simulations show the feasibility of the proposed approach. As a benchmarking topology at the working frequency of $35.4 \mathrm{GHz}$, an Archimedean spiral antenna matched to a heterogeneous transformer, which couples the power received by the antenna to the chip, has been simulated. Transistor level circuit simulations are also proposed for the LNA and the detector, which together will constitute the system-on-chip (SoC) radiometer to be integrated in the LCP-SoP.
\end{abstract}

Index Terms - Electromagnetic coupling, flexible electronics, heterogeneous integration, SoC, SoP, LNA, imaging, mm-wave.

\section{INTRODUCTION}

To afford the complex assembly issues related to large arrays of radiometric receivers [1], this paper proposes a new approach based on the chip-to-package wireless power transfer. In particular, a $35.4 \mathrm{GHz}$ System-in-Package (SiP) radiometer is considered as case-of-study. A standard $250 \mathrm{~nm}$ SiGe BiCMOS technology has been accounted for in the chip design. The planar antenna is designed to be fabricated on a flexible liquid crystal polymer (LCP) substrate. The RF connection between the MMIC and the antenna is realized in a contactless approach [2], thus avoiding the galvanic connection, usually made by vias or bond wires, which at millimeter wave could generate many parasitic effects and increase the cost, while suffering from potential microfracturing issues for extreme curvature radii. The substrate material can be stacked up in a multilayer lamination process in such a way as to serve as a package for the system.

\section{SYSTEM DESCRIPTION}

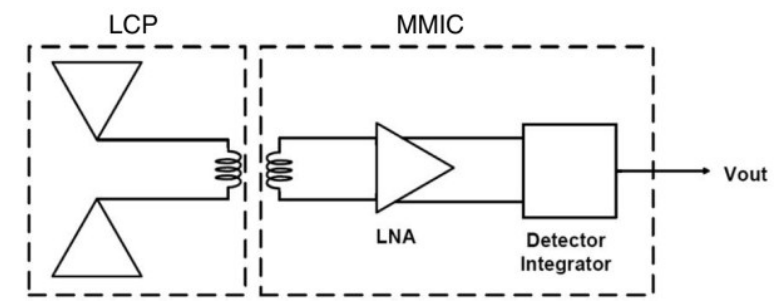

Fig. 1. System diagram.

The system is designed to work at the center frequency of $35.4 \mathrm{GHz}$, in the Ka-band frequency window where the atmospheric attenuation is found to be relatively low, and thus suitable for the imaging applications described in the previous paragraph. The essential principle of the operation of the system is the magnetic coupling between the antenna and the chip, as shown in Fig. 1. Such a contactless coupling is obtained by a heterogeneous transformer, the primary and secondary windings of which are fabricated on the LCP substrate and Si chip respectively. The chip is a directdetection total power radiometer. It consists of the secondary winding of the heterogeneous transformer, the low-noise amplifier (LNA) and the detector/integrator. Substantially, the secondary coil of the transformer will substitute the input pads of the chip. The DC voltage detected by the radiometer is then passed through the side of the flexible substrate using wire bonds, as shown in Fig. 2. Multiple layers of thin-film LCP can be arranged and laminated to form the packaging structure required. The base layers for the stackup are the RO3850 and RO3908 LCP sheets from Rogers Corporation. Low melting temperature layers (RO3908) are used to adhere/stick thicker high melting temperature core layers (RO3850) in order to create a homogeneous LCP package stackup similar to the one shown in Fig. 2. Metal 1 will be used for the antenna, the matching network and the primary coil. The top on-chip metal will be used for the secondary coil. Metal 0 will be used for the ground plane.

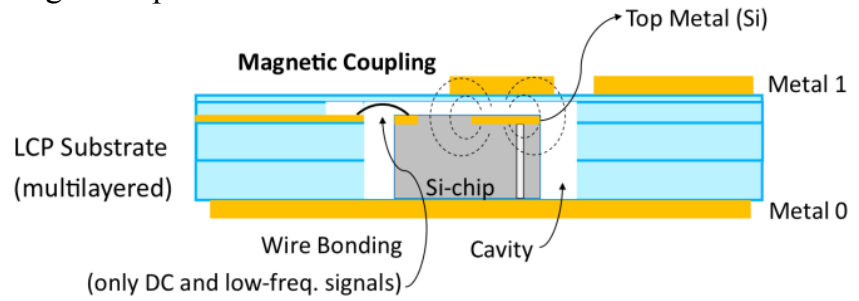

Fig. 2. Side view of the package stackup.

\section{EM SIMULATIONS RESULTS}

In this section, the proposed idea is validated by means of full-wave EM simulations. A heterogeneous transformer between the LCP substrate and BiCMOS Si chip has been designed and simulated. The geometry of the transformer has been defined on the basis of the resolution capabilities of the LCP fabrication facilities, which impose the lower limit on primary coil diameter and thus on the whole transformer dimensions. The two coils are separated by a distance of 25 $\mu \mathrm{m}$ and have been placed face-to-face. For the secondary coil, 
a $250 \mathrm{~nm} \mathrm{SiGe} \mathrm{BiCMOS}$ process has been taken into account, using the available thick top metal layers for the coil and underpass design. The transformer is shown in Fig. 3 and its dimensions are summarized in Tab. I.

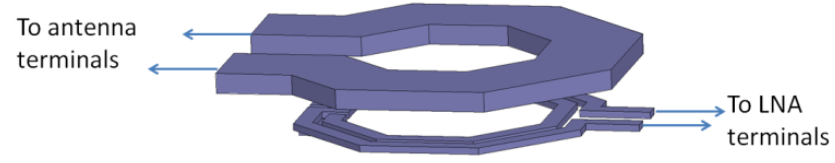

Fig. 3. Heterogeneous transformer. The upper coil (primary) is built on Metal 1 (see Fig. 2) and the lower coil (secondary) is built on chip using the topmost metal available in the BiCMOS process.

The obtained maximum available power gain (MAG) of the transformer is $-0.66 \mathrm{~dB}$ at $35.4 \mathrm{GHz}$, thus indicating that, with the right matching, only $14 \%$ of the incident power is lost inside the transformer.

TABLE I

DIMENSIONS OF THE HETEROGENEOUS TRANSFORMER

\begin{tabular}{|c|c|c|c|c|c|}
\hline & $\begin{array}{c}\mathrm{D} \\
{[\mu \mathrm{m}]}\end{array}$ & $\begin{array}{c}\text { Width } \\
{[\mu \mathrm{m}]}\end{array}$ & $\begin{array}{c}\text { Spacing } \\
{[\mu \mathrm{m}]}\end{array}$ & $\begin{array}{c}\mathrm{N} \\
\text { turns }\end{array}$ & $\begin{array}{c}\text { Thick. } \\
{[\mu \mathrm{m}]}\end{array}$ \\
\hline Primary & 220 & 50 & 50 & 1 & 9 \\
\hline Secondary & 160 & 8 & 2 & 2 & 3 \\
\hline
\end{tabular}

The two-wire Archimedean spiral configuration has been chosen for the antenna design due to its balance feed terminal and circular polarization. Additionally, the Archimedean configuration with the reflector allows the spiral antennas to achieve high gain with radiation pattern of a broadside antenna. Fig. 4a shows the top view of the designed antenna. The secondary coil of the transformer, buried below the visible layer in figure (as shown in Fig. 3), couples the RF signal received by the antenna to the chip. The antenna reflector is made of the Metal 0 layer shown in Fig. 2.

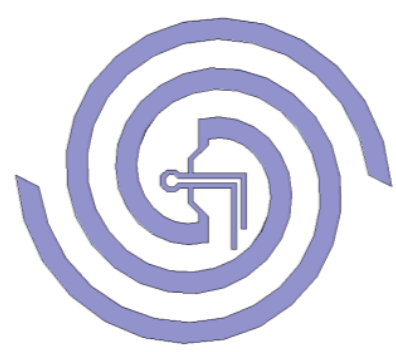

(a)

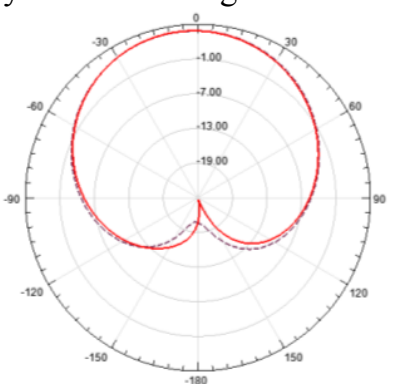

(b)
Fig. 4. (a) Top view of the designed antenna (lying on the $x-y$ plane) and (b) its radiation pattern. E-plane ( $x-z$ plane) in solid line, H-plane ( $y$-z plane) in dashed line.

A feed network based on microstrip line is designed for the spiral antenna to allow maximum power transfer from the antenna to the coils and ultimately the chip. This network is constituted by the high-impedance connections and by the two open stubs (balanced structure) shown again in the layout of Fig. 4a. The simulation port has been placed at the terminals of the secondary coil. The total gain of the structure from simulations is about $5 \mathrm{~dB}$ at $35.4 \mathrm{GHz}$. The radiation a pattern is shown in Fig. $4 \mathrm{~b}$ and the input reflection coefficient (S11) is shown in Fig. 5 respectively illustrating the directive radiation of the design and excellent contactless power transfer.

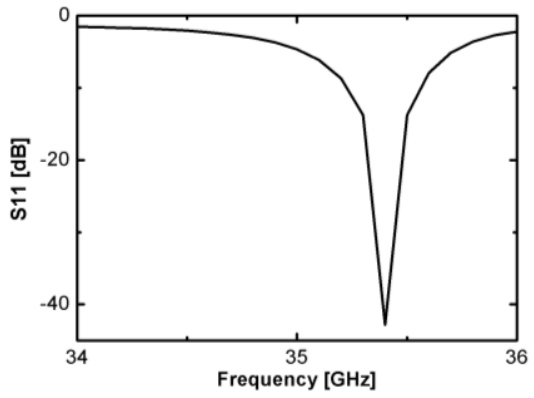

Fig. 5. Input reflection coefficient of the antenna shown in Fig. 4. The simulation port has been placed at the terminals of the secondary coil.

\section{TRANSISTOR LEVEL SIMULATIONS RESULtS}

In this section a $250 \mathrm{~nm}$ SiGe BiCMOS technology, developed at the IHP foundry, is exploited for the circuit design. Thanks to the use of an automatic procedure purposely developed [3], we were able to consider all the integrated inductors with their rigorous electromagnetic models.

The LNA is shown in Fig. 6a. It employs three differential cascode stages. For simultaneous noise and input power matching, we used a well-known LNA design procedure: we adjusted the emitter length to set the optimal noise resistance at the optimal current density. Then, the degeneration inductors $\left(\mathrm{L}_{\mathrm{E}}\right.$ in Fig. 6a) increase the input resistance to $106 \Omega$ (as resulted from the EM simulations discussed in the previous section). Finally the base inductors ( $\mathrm{L}_{\mathrm{IN}}$ in Fig. 6a) match the input reactance to $3.12 \Omega$. The bias circuitry is based on a cascode current mirror optimized to keep the gain stable over temperature changes.

The schematic of the designed HBTs detector is shown in Fig. 6b. It is a full differential architecture composed by coupled common emitter transistors forming the detector and by a dummy detector in perfect layout symmetry. The dummy detector provides an output reference voltage for the main detector output to be used with a differential post-amplifier. The HBTs are biased through a resistive network and the signal from the LNA is applied differentially to the bases of $\mathrm{Q}_{1,2}$. The DC output voltage is taken at the common collector point. Table II shows the results of the simulations.

TABLE II

Simulations RESUlTS AT $35.4 \mathrm{GHz}$

\begin{tabular}{|c|c|c|c|c|c|c|}
\hline \multicolumn{4}{|c|}{ LNA } & \multicolumn{2}{|c|}{ Detector, -32 dBm in. pow. } \\
\hline $\begin{array}{c}\text { Gain } \\
{[\mathrm{dB}]}\end{array}$ & $\begin{array}{c}\mathrm{NF} \\
{[\mathrm{dB}]}\end{array}$ & $\begin{array}{c}\mathrm{S}_{11} / \mathrm{S}_{22} \\
{[\mathrm{~dB}]}\end{array}$ & $\begin{array}{c}\mathrm{P}_{\mathrm{DC}} \\
{[\mathrm{mW}]}\end{array}$ & $\begin{array}{c}\text { Resp. } \\
{[\mathrm{mV} / \mu \mathrm{W}]}\end{array}$ & $\begin{array}{c}\mathrm{NEP} \\
{[\mathrm{pW} / \sqrt{\mathrm{Hz}}]}\end{array}$ & $\begin{array}{c}\mathrm{P}_{\mathrm{DC}} \\
{[\mathrm{mW}]}\end{array}$ \\
\hline 40.5 & 4.5 & $-40 /-44$ & 95 & 96 & 5.1 & 3.75 \\
\hline
\end{tabular}




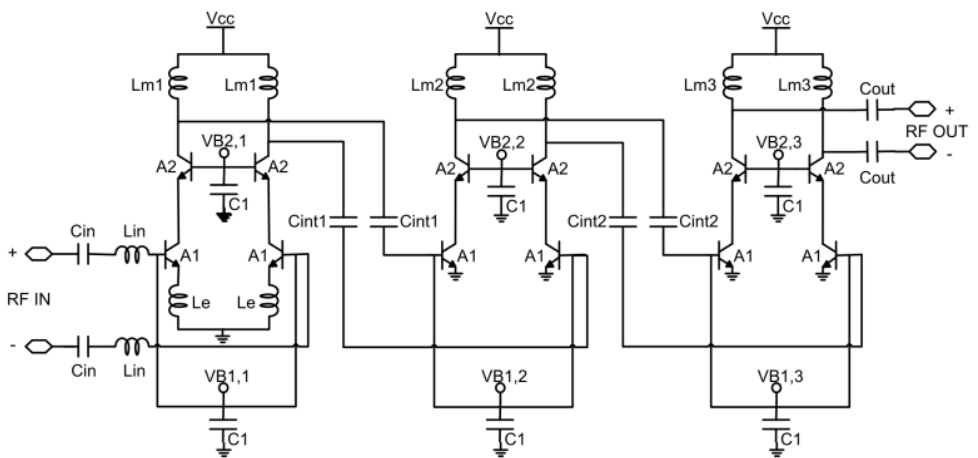

(a)

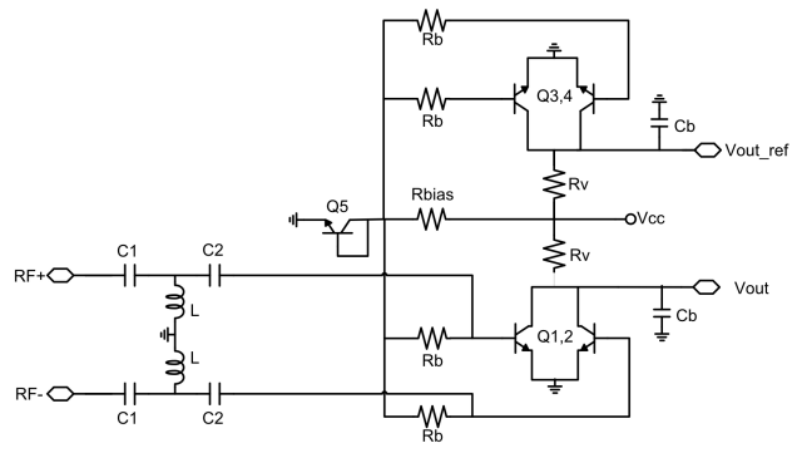

(b)

Figure 6. Schematics of the $35.4 \mathrm{GHz}$ differential circuits designed. (a) Schematic of the LNA and (b) Schematic of the detector. For the simulations of the two circuits we used Agilent ADS S-PAR (for the LNA) and Harmonic Balance (for the detector) solvers.

\section{CONCLUSION}

Chip-to-package wireless power transfer has been applied to antennas and MMICs on LCP flexible substrate. Millimeterwave operation is introduced and validated with EM simulations. The circular polarization gain of the antenna and the S11 level achieved show that the proposed approach enables a significant reduction in the manufacturing cost of imaging sensors simplifying the production while enhancing the interconnect ruggedness for conformal geometries. Then a purposely designed MMIC to form the complete imaging sensor is designed and simulated in a $250 \mathrm{~nm}$ SiGe technology showing the readiness of the selected technology for this new approach. The SoC radiometer will allow the innovative integration of each sensor's front-end with the respective antenna, constituting a single element of the array. Thanks to this a considerable reduction in the size, cost and power consumption of the system can be achieved.

\section{APPENDIX I}

\section{MEASUREMENTS RESULT AT $1.5 \mathrm{GHz}$}
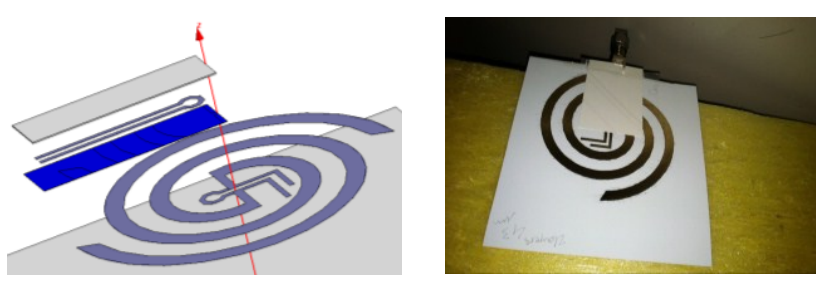

Fig. 7. (a) exploded view of the fabricated prototype. (b) capture of the fabricated prototype at $1.5 \mathrm{GHz}$ range.

To validate the concept of contactless coupling of the coils and the power collecting capability at a given frequency band of the proposed design, a prototype was fabricated by inkjet printing on paper substrates and measured. Fig. 7a shows an exploded view of the measured prototype. The top layer is a paper superstrate with thickness 220 um below which is built the secondary (1-turn) coil including the input lines. Below is the glue layer (in blue). A super glue layer having dielectric constant of 3.3 and loss tangent of 0.06 with the estimated thickness of $100 \mu \mathrm{m}$ was utilized to attach the superstrate to the substrate on which the antenna and the primary coil are built. The final assembled prototype is shown in Fig. 7b.

The magnetic coupling has been implemented by a homogeneous transformer having both primary and secondary coil on flexible substrate. The simulated maximum available power gain (MAG) of the transformer is $-0.1 \mathrm{~dB}$ at $1.5 \mathrm{GHz}$, thus indicating that, with the right matching, only $3 \%$ of the incident power is lost inside the transformer. The measurement results are shown in Fig. 8, giving the $|\mathrm{S} 11|$ of $12 \mathrm{~dB}$ at $1.5 \mathrm{GHz}$. The slight shift in the measurement is due to the instrument uncertainty in the glue thickness estimation.

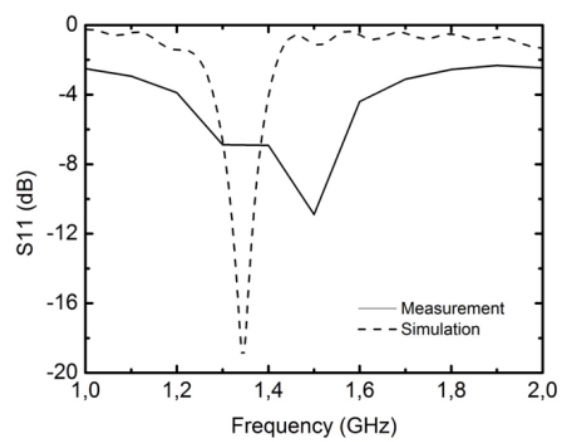

Fig. 8. Measured (solid-line) and simulated (dashed-line) S11 of the prototype antenna shown in Fig. $7 \mathrm{~b}$.

\section{REFERENCES}

[1] V. Gorishnyak, et.al, " $8 \mathrm{~mm}$ Passive Imaging System with 32 Sensors," IEEE EURAD 2004, pp.333-336, October 2004.

[2] F. Alimenti, et.al, "A New Contactless Assembly Method for Paper Substrate Antennas and UHF RFID Chips," IEEE MTTTrans., vol.59, no.3, pp.627-637, March 2011.

[3] L. Aluigi, et.al, "Automatic Design and 3D Electromagnetic Simulation of sub-nH Spiral Inductors," Proceedings of PIERS, Marrakesh, pp. 1719-1722, March 2011. 